PROCEEDINGS OF THE

AMERICAN MATHEMATICAL SOCIETY

Volume 139, Number 5, May 2011, Pages 1889-1896

S 0002-9939(2010)10617-2

Article electronically published on October 29, 2010

\title{
SLOPES AND COLORED JONES POLYNOMIALS OF ADEQUATE KNOTS
}

\author{
DAVID FUTER, EFSTRATIA KALFAGIANNI, AND JESSICA S. PURCELL
}

(Communicated by Daniel Ruberman)

\begin{abstract}
Garoufalidis conjectured a relation between the boundary slopes of a knot and its colored Jones polynomials. According to the conjecture, certain boundary slopes are detected by the sequence of degrees of the colored Jones polynomials. We verify this conjecture for adequate knots, a class that vastly generalizes that of alternating knots.
\end{abstract}

\section{INTRODUCTION}

For a knot $K \subset S^{3}$, let $N_{K}$ denote a tubular neighborhood of $K$ and let $M_{K}:=$ $\overline{S^{3} \backslash N_{K}}$ denote the exterior of $K$. Let $\langle\mu, \lambda\rangle$ be the canonical meridian-longitude basis of $H_{1}\left(\partial N_{K}\right)$. An element $p / q \in \mathbb{Q} \cup\{1 / 0\}$ is called a boundary slope of $K$ if there is a properly embedded essential surface $(S, \partial S) \subset\left(M_{K}, \partial N_{K}\right)$, such that every circle of $\partial S$ is homologous to $p \mu+q \lambda \in H_{1}\left(\partial N_{K}\right)$. Hatcher has shown that every knot $K \subset S^{3}$ has finitely many boundary slopes [7].

The colored Jones function of $K$ is a sequence of Laurent polynomial invariants $J_{K}(n, q) \in \mathbb{Z}\left[q, q^{-1}\right]$, for $n=1,2, \ldots$ For $n=2, J_{K}(2, q)$ is the ordinary Jones polynomial. We will use the normalization that $J_{\text {unknot }}(n, q)=1$, for every $n \geq 1$. Let $j(n)$ denote the highest degree of $J_{K}(n, q)$ in $q$, and let $j^{*}(n)$ denote the lowest degree. Consider the sequences

$$
j s_{K}:=\left\{\frac{4 j(n)}{n^{2}}: n>0\right\} \quad \text { and } \quad j s_{K}^{*}:=\left\{\frac{4 j^{*}(n)}{n^{2}}: n>0\right\} .
$$

Garoufalidis conjectured [6] that for each knot $K$, every cluster point (i.e., every limit of a subsequence) of $j s_{K}$ or $j s_{K}^{*}$ is a boundary slope of $K$. Thus, if the conjecture holds, the colored Jones polynomials detect certain boundary slopes of $K$. He verified the conjecture for alternating knots, torus knots, pretzel knots of type $(-2,3, p)$, and several low-crossing knots.

In this paper, we prove Garoufalidis' conjecture for the class of adequate knots. The precise definition of adequate appears in Section 2, For the moment, we note that the family of adequate knots includes all alternating knots, most Montesinos knots, and all knots that are Conway sums of two strongly alternating tangles. See [10] and Section 4 for more examples.

Received by the editors February 8, 2010 and, in revised form, May 25, 2010.

2010 Mathematics Subject Classification. Primary 57M25, 57M27.

The first author is supported in part by NSF grant DMS-1007221.

The second author is supported in part by NSF grant DMS- 0805942 .

The third author is supported in part by NSF grant DMS-0704359. 
Theorem 1. Let $D(K)$ be a knot diagram. Then

(a) If $D$ is $A$-adequate, then $\lim _{n \rightarrow \infty} 4 n^{-2} j^{*}(n)$ exists and is a boundary slope of $K$.

(b) If $D$ is $B$-adequate, then $\lim _{n \rightarrow \infty} 4 n^{-2} j(n)$ exists and is a boundary slope of $K$.

In particular, if $K$ is a non-trivial adequate knot, then the set $j s_{K} \cup j s_{K}^{*}$ has exactly two cluster points, both of which are integer boundary slopes of $K$.

The proof of Theorem 1 involves four steps:

(1) Starting with an $A$-adequate diagram $D(K)$, construct a state surface $S_{A}$ whose boundary is $K$. This is a standard construction, generalizing the construction of a checkerboard surface.

(2) Verify that $S_{A}$ is an essential surface. This result, stated as Theorem 3 below, was first proved by Ozawa [11; an alternate proof is given by the authors in [4].

(3) Relate the boundary slope of $S_{A}$ to the number of positive and negative crossings in the diagram $D$. This is carried out in Lemma 5 .

(4) Relate the limit of $j s_{K}^{*}$ to the number of positive and negative crossings in the diagram $D$. This is carried out in Lemma 6.

Taken together, Theorem 3 and Lemmas 5 and 6 immediately imply Theorem 1 (a). Part (b) of the theorem follows by considering the mirror image of the diagram $D$.

\section{StATE GRAPHS AND SURFACES}

Let $D$ be a link diagram, and $x$ a crossing of $D$. Associated to $D$ and $x$ are two link diagrams, each with one fewer crossing than $D$, called the $A$-resolution and $B-$ resolution of the crossing. See Figure 1

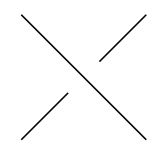

Figure $1 . A$ - and $B$-resolutions of a crossing.

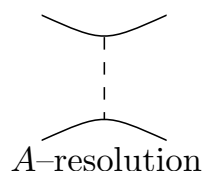

$A$-resolution

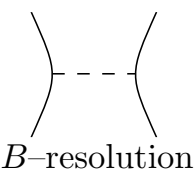

$B$-resolution

A Kauffman state $\sigma$ is a choice of $A$-resolution or $B$-resolution at each crossing of $D$. Corresponding to every state $\sigma$ is a crossing-free diagram $s_{\sigma}$ : this is a collection of circles in the projection plane. We can encode the choices that led to the state $\sigma$ in a graph $\mathbb{G}_{\sigma}$, as follows. The vertices of $\mathbb{G}_{\sigma}$ are in $1-1$ correspondence with the state circles of $s_{\sigma}$. Every crossing $x$ of $D$ corresponds to a pair of arcs that belong to circles of $s_{\sigma}$; this crossing gives rise to an edge in $\mathbb{G}_{\sigma}$ whose endpoints are the state circles containing those arcs.

Every Kauffman state $\sigma$ also gives rise to a surface $S_{\sigma}$, as follows. Each state circle of $\sigma$ bounds a disk in $S^{3}$. This collection of disks can be disjointly embedded in the ball below the projection plane. At each crossing of $D$, we connect the pair of neighboring disks by a half-twisted band to construct a surface $S_{\sigma} \subset S^{3}$ whose boundary is $K$. See Figure 2 for an example where $\sigma$ is the all $-A$ state.

Well-known examples of state surfaces include Seifert surfaces (where the corresponding state $\sigma$ is defined by following an orientation on $K$ ) and checkerboard 

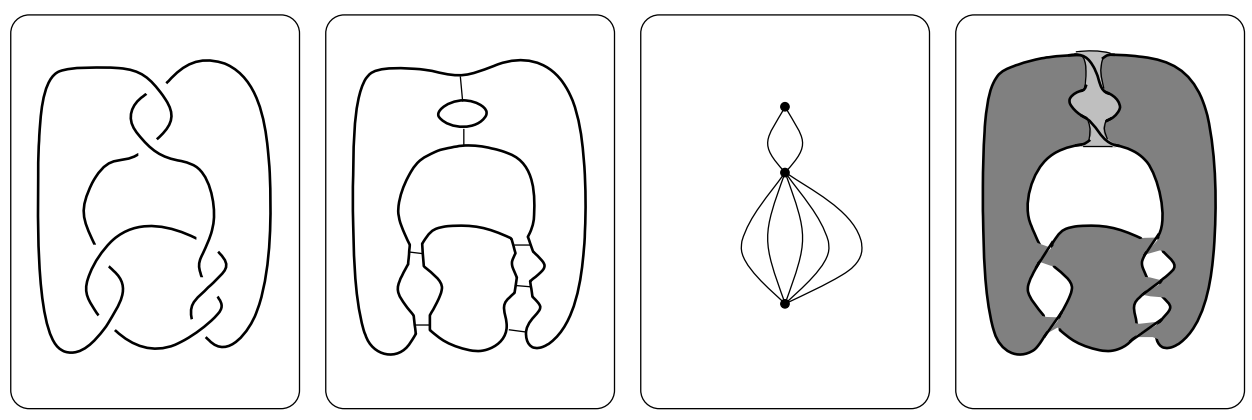

Figure 2. Left to right: A diagram. The diagram with $A-$ resolutions at each crossing. The graph $\mathbb{G}_{A}$. State surface $S_{A}$.

surfaces for alternating links (where the corresponding state $\sigma$ is either the all- $A$ or all $-B$ state). In this paper, we focus on the all $-A$ and all $-B$ states of a diagram, but we do not require our diagrams to be alternating. Thus our surfaces are generalizations of checkerboard surfaces.

Definition 2. A link diagram $D$ is called $A$-adequate if the state graph $\mathbb{G}_{A}$ corresponding to the all $-A$ state contains no 1 -edge loops. Similarly, $D$ is called $B$-adequate if the all- $B$ graph $\mathbb{G}_{B}$ contains no 1 -edge loops. A link diagram is adequate if it is both $A$ - and $B$-adequate. A link that admits an adequate diagram is also called adequate.

Adequate diagrams are quite common. All reduced alternating diagrams are adequate. Every $n$-string planar cable of an adequate diagram is adequate. The standard diagram of a Montesinos link is either $A$-adequate or $B$-adequate, and typically both. Finally, observe that a diagram $D(K)$ is $A$-adequate if and only if its mirror image is $B$-adequate. This observation is useful for the proofs below: once a result is proved for the all- $A$ state surface $S_{A}$ of an $A$-adequate diagram, the corresponding statement about $B$-adequate diagrams follows by reflection.

Theorem 3 (Ozawa 11). Let $D$ be an A-adequate diagram of a knot K. Then the state surface $S_{A}$ is incompressible and $\partial$-incompressible in the complement $M_{K}$. Similarly, if $D$ is a $B$-adequate diagram of a knot $K$, then $S_{B}$ is incompressible and $\partial$-incompressible.

Ozawa's original proof of this theorem relies on building up the surface $S_{\sigma}$ via Murasugi sums. An alternate proof from the point of view of normal surface theory will be given by the authors in [4, where we will also relate the coefficients of the colored Jones polynomials $J_{K}(n, q)$ to the size of the guts of the surfaces $S_{A}$ and $S_{B}$. These guts can be viewed as the hyperbolic pieces in the geometric decomposition of $S^{3} \backslash S_{\sigma}$. Thus, taken together, 4 and Theorem 1 of this paper establish two separate connections between the colored Jones polynomials and classical geometric topology.

Recall from the Introduction that if $S \subset M_{K}$ is a surface such that $\partial S$ represents the homology class $p \mu+q \lambda \in H_{1}\left(\partial M_{K}\right)$, we say that the boundary slope of $S$ is $p / q \in \mathbb{Q} \cup\{\infty\}$. It turns out that the boundary slope of a state surface $S_{\sigma}$ is easy to read off from a diagram $D$. 
Suppose that $D(K)$ is a diagram of an oriented knot $K$. Then every crossing of $D$ can be classified as either positive or negative, as in Figure 3. For a state $\sigma$ of $D$, let $c_{+}^{B}(\sigma)$ be the number of positive crossings at which $\sigma$ chooses the $B$-resolution. Similarly, let $c_{-}^{A}(\sigma)$ be the number of negative crossings at which $\sigma$ chooses the $A$-resolution.

Lemma 4. Let $D(K)$ be a diagram of an oriented knot $K$, and let $\sigma$ be a state of $D$. Then the state surface $S_{\sigma}$ has as its boundary the slope $2 c_{+}^{B}(\sigma)-2 c_{-}^{A}(\sigma)$.

This lemma was observed by Curtis and Taylor for checkerboard surfaces of alternating knots [1, Proposition 2.6]. However, both the statement and the proof hold in complete generality: $S_{\sigma}$ is not even required to be an essential surface.
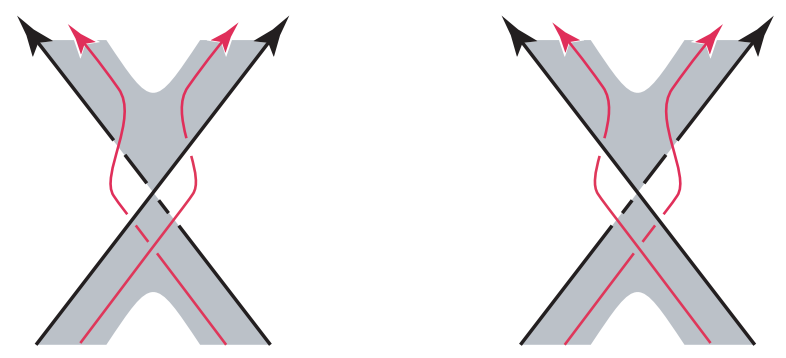

FIGURE 3. Left: a positive crossing, and a piece of state surface $S_{\sigma}$ that chooses the $B$-resolution at this crossing. Locally, this crossing contributes +2 to the slope of $S_{\sigma}$. Right: if $\sigma$ chooses the $A$-resolution at a negative crossing, the slope of $S_{\sigma}$ receives a local contribution of -2 .

Proof. Suppose, first, that $\sigma$ is the Seifert state and that $S_{\sigma}$ is an oriented Seifert surface constructed from the diagram $D$. To follow an orientation of $K, \sigma$ must choose the $A$-resolution at every positive crossing and the $B$-resolution at every negative crossing (the opposite of the choices depicted in Figure 3). Thus $c_{+}^{B}(\sigma)=$ $c_{-}^{A}(\sigma)=0$, by definition. Also, because $\partial S_{\sigma}$ is the boundary of an orientable surface, this curve is null-homologous in $M_{K}$ and has slope 0 . This verifies the lemma for the Seifert state.

Next, let $\sigma$ be an arbitrary state. Then it is still the case that $S_{\sigma}$ intersects a meridian of $K$ only once. Thus the boundary slope of $S_{\sigma}$ is still an integer $p$. Let $L$ be the simple closed curve of intersection between $S_{\sigma}$ and $\partial M_{K}$, oriented in the same direction as $K$. Then the boundary slope $p$ of $S_{\sigma}$ is the linking number $l k(K, L)$, or equivalently the oriented intersection number between $L$ and a Seifert surface for $K$.

The linking number $l k(K, L)$ can be computed by summing the local contributions near each crossing. If $\sigma$ chooses the $A$-resolution at a positive crossing or the $B$-resolution at a negative crossing, $L$ is locally disjoint from the Seifert surface, and the local contribution to the linking number is 0 . On the other hand, for every positive crossing where $\sigma$ chooses the $B$-resolution, the left panel of Figure 3 shows that the neighborhood of the crossing contributes +2 to the linking number $l k(K, L)$. Similarly, the right panel of Figure 3 shows that a negative crossing where $\sigma$ chooses the $A$-resolution contributes -2 to $l k(K, L)$.

Adding up these contributions, we conclude that $l k(K, L)=2 c_{+}^{B}(\sigma)-2 c_{-}^{A}(\sigma)$. 
As a special case of Lemma 4, we obtain the boundary slopes of $S_{A}$ and $S_{B}$.

Lemma 5. Let $D(K)$ be a diagram of an oriented knot $K$. Let $c_{+}$be the number of positive crossings in $D$, and $c_{-}$the number of negative crossings. Then the all- $A$ surface $S_{A}$ has boundary slope $-2 c_{-}$, and $S_{B}$ has boundary slope $2 c_{+}$.

Proof. The all $-A$ state $\sigma$ chooses the $A$-resolution at every crossing. Thus for the all- $A$ state, $c_{+}^{B}(\sigma)=0$ and $c_{-}^{A}(\sigma)=c_{-}$; hence $\partial S_{A}$ has slope $-2 c_{-}$by Lemma 4 . Similarly, for the all- $B$ state $\sigma, c_{+}^{B}(\sigma)=c_{+}$and $c_{-}^{A}(\sigma)=0$; hence $\partial S_{B}$ has slope $2 c_{+}$.

\section{Colored Jones polynomials}

In this section, we relate the degrees of colored Jones polynomials to the numbers $c_{+}$and $c_{-}$of positive and negative crossings in a diagram $D$. A good reference for the following discussion is Lickorish's book 9 .

The colored Jones polynomials of a link $K$ have a convenient expression in terms of Chebyshev polynomials. For $n \geq 0$, the polynomial $S_{n}(x)$ is defined recursively as follows:

$$
S_{n+1}=x S_{n}-S_{n-1}, \quad S_{1}(x)=x, \quad S_{0}(x)=1 .
$$

Let $D$ be a diagram of a link $K$. For an integer $m>0$, let $D^{m}$ denote the diagram obtained from $D$ by taking $m$ parallel copies of $K$. This is the $m$-cable of $D$ using the blackboard framing; if $m=1$, then $D^{1}=D$. Let $\left\langle D^{m}\right\rangle$ denote the Kauffman bracket of $D^{m}$ : this is a Laurent polynomial over the integers in a variable $A$. Let $w=w(D)=c_{+}-c_{-}$denote the writhe of $D$. Then we may define the function

$$
G(n+1, A):=\left((-1)^{n} A^{n^{2}+2 n}\right)^{-w}(-1)^{n-1}\left(\frac{A^{4}-A^{-4}}{A^{2 n}-A^{-2 n}}\right)\left\langle S_{n}(D)\right\rangle,
$$

where $S_{n}(D)$ is a linear combination of blackboard cablings of $D$, obtained via equation (1), and the notation $\left\langle S_{n}(D)\right\rangle$ means to extend the Kauffman bracket linearly. That is, for diagrams $D_{1}$ and $D_{2}$ and scalars $a_{1}$ and $a_{2},\left\langle a_{1} D_{1}+a_{2} D_{2}\right\rangle=$ $a_{1}\left\langle D_{1}\right\rangle+a_{2}\left\langle D_{2}\right\rangle$. For the results below, the important corollary of the recursive formula for $S_{n}(x)$ is that

$$
S_{n}(D)=D^{n}+(1-n) D^{n-2}+\text { lower degree cablings of } D \text {. }
$$

Finally, the reduced $(n+1)$-colored Jones polynomial of $K$, denoted by $J_{K}(n+$ $1, q)$, is obtained from $G(n+1, A)$ by substituting $q:=A^{-4}$.

Recall from the Introduction that $j(n)$ denotes the highest degree of $J_{K}(n, q)$ in $q$, and $j^{*}(n)$ denotes the lowest degree. Following the big- $O$ notation, we let $O(n)$ denote a term that is at most linear in $n$.

Lemma 6. Let $D$ be a link diagram with $c_{+}$positive crossings and $c_{-}$negative crossings.

(a) If $D$ is $A$-adequate, then $j^{*}(n)=-\frac{c_{-}}{2} n^{2}+O(n)$.

(b) If $D$ is $B$-adequate, then $j(n)=\frac{c_{+}}{2} n^{2}+O(n)$.

Proof. For part (a), let $D$ be an $A$-adequate diagram with $c=c(D)=c_{+}+c_{-}$ crossings. Let $v_{A}=v_{A}(D)$ be the number of vertices of $\mathbb{G}_{A}$, which is equal to the 
number of state circles in the all- $A$ state. Then, for every $m>0$, the link diagram $D^{m}$ is also $A$-adequate, with $c\left(D^{m}\right)=m^{2} c$ and $v_{A}\left(D^{m}\right)=m v_{A}$.

Let $\operatorname{deg}(P(A))$ denote the highest degree of a polynomial $P$ in $A$. Then the highest degree of $\langle D\rangle$ is $\operatorname{deg}\langle D\rangle=c+2 v_{A}-2$; see [9, Lemma 5.4] or [2, Lemma 7.1] for a proof. From equation (2), one can see that $\operatorname{deg}(G(n, A))$ comes from the highest-degree term of $\left\langle S_{n-1}(D)\right\rangle$. Furthermore, by equation (3) and the previous paragraph,

$$
\operatorname{deg}\left\langle S_{n-1}(D)\right\rangle=\operatorname{deg}\left\langle D^{n-1}\right\rangle=(n-1)^{2} c+2(n-1) v_{A}-2 .
$$

Thus

$$
\begin{aligned}
\operatorname{deg} G(n, A) & =-w\left(n^{2}+2 n\right)+(4-2 n)+\operatorname{deg}\left\langle S_{n-1}(D)\right\rangle \\
& =-w\left(n^{2}+2 n\right)+(4-2 n)+(n-1)^{2} c+2(n-1) v_{A}-2 \\
& =(c-w) n^{2}+O(n) \\
& =\left(\left(c_{+}+c_{-}\right)-\left(c_{+}-c_{-}\right)\right) n^{2}+O(n) \\
& =2 c_{-} n^{2}+O(n) .
\end{aligned}
$$

Finally, since $J_{K}(n, q)$, is obtained from $G(n, A)$ by substituting $q:=A^{-4}$, we conclude that the lowest degree of $J_{K}(n, q)$ in $q$ is $j^{*}(n)=-c_{-} n^{2} / 2+O(n)$. This proves (a).

For part (b), it suffices to observe that the mirror image $D^{*}$ of a $B$-adequate diagram $D$ will be $A$-adequate. Taking the mirror image also interchanges positive and negative crossings, and replaces $q$ with $q^{-1}$ in the colored Jones polynomials. Thus the result follows from (a).

We can now complete the proof of Theorem 1.

Proof of Theorem 1. Let $D(K)$ be an $A$-adequate diagram. Then, by Theorem 3 $S_{A}$ is an essential surface for $M_{K}$. By Lemmas 5 and 6 , the boundary slope of $S_{A}$ is

$$
-2 c_{-}=\lim _{n \rightarrow \infty} 4 n^{-2} j^{*}(n) .
$$

Similarly, if $D(K)$ is $B$-adequate, $S_{B}$ is an essential surface with boundary slope

$$
2 c_{+}=\lim _{n \rightarrow \infty} 4 n^{-2} j(n) .
$$

In particular, if $D(K)$ is adequate, then the sequences $\left\{4 n^{-2} j^{*}(n)\right\}$ and $\left\{4 n^{-2} j(n)\right\}$ both converge to boundary slopes of $K$. For a non-trivial knot $K$, the slopes $-2 c_{-}$ and $2 c_{+}$are distinct, because at least one of the integers $c_{-}$and $c_{+}$is strictly positive.

\section{EXAmples}

Example 7. Let $p$ be an odd integer, and let $K_{p}$ denote the $(-2,3, p)$ pretzel knot, with a standard pretzel diagram $D_{p}$. It is easy to check that $D_{p}$ is $A$-adequate iff $p>0$ and $B$-adequate iff $p<0$. Furthermore, for all values of $p$ except $p=-1$ (when $D_{p}$ is an unusual diagram of the $5_{2}$ knot), the knot $K_{p}$ does not admit an adequate diagram. This classical fact can also be seen via Theorem 1, because all Jones slopes of adequate knots are integers. 
Garoufalidis computed [6] that

$$
\lim _{n \rightarrow \infty} \frac{4 j(n)}{n^{2}}=\frac{2\left(p^{2}-p-5\right)}{p-3} \text { if } p \geq 5, \quad \lim _{n \rightarrow \infty} \frac{4 j^{*}(n)}{n^{2}}=\frac{2(p+1)^{2}}{p} \quad \text { if } p \leq-3 .
$$

From the work of Hatcher and Oertel $[8$ and Dunfield [3], it follows that these limiting numbers are indeed boundary slopes of $K_{p}$. Hence, since all Jones slopes of adequate knots are integers by Theorem 1, we recover the classical fact that these knots are not adequate.

Example 8. Following [10, a $(2,2)$-tangle $T$ is called strongly alternating if each of the closures of $T$ is a reduced alternating link diagram. Any knot obtained as a Conway sum of two strongly alternating tangles is then adequate (see also [5]). For example, any non-alternating pretzel knot $K\left(a_{1}, \ldots, a_{r}, b_{1}, \ldots, b_{k}\right)$ with $a_{i}, b_{j}, r, k \geq 2$ is adequate. Similarly, as explained in [10, a reduced diagram of any Montesinos knot with at least two positive rational tangles and at least two negative rational tangles will be adequate. Theorem 1 implies that these knots satisfy Garoufalidis' conjecture.

Example 9. Let $B_{n}$ denote the braid group on $n$ strings, and let $\sigma_{1}, \ldots, \sigma_{n-1}$ be the elementary braid generators. Let $D_{b}$ denote the closed braid diagram obtained from the braid $b=\sigma_{i_{1}}^{r_{1}} \sigma_{i_{2}}^{r_{2}} \cdots \sigma_{i_{k}}^{r_{k}}$. If $r_{j}>0$ for all $j$, the positive braid diagram $D_{b}$ will be $A$-adequate. Since all crossings in this braid are positive, $c_{-}=0$. Thus, by Lemma 6

$$
\lim _{n \rightarrow \infty} 4 n^{-2} j^{*}(n)=-2 c_{-}=0 .
$$

Furthermore, the essential surface $S_{A}$ whose boundary is this slope will be a fiber in $S^{3} \backslash K$.

Under the stronger hypothesis that $r_{j} \geq 3$ for all $j$, the diagram $D_{b}$ is not only $A$-adequate but also $B$-adequate. Thus Theorem 1 applies. The other boundary slope detected by the colored Jones polynomials is

$$
\lim _{n \rightarrow \infty} 4 n^{-2} j(n)=2 c_{+}=2 \sum_{j=1}^{k} r_{j} .
$$

\section{REFERENCES}

[1] Cynthia L. Curtis and Samuel Taylor, The Jones polynomial and boundary slopes of alternating knots, arXiv:0910.4912.

[2] Oliver T. Dasbach, David Futer, Efstratia Kalfagianni, Xiao-Song Lin, and Neal W. Stoltzfus, The Jones polynomial and graphs on surfaces, Journal of Combinatorial Theory Ser. B 98 (2008), no. 2, 384-399. MR2389605 (2009d:57020)

[3] Nathan M. Dunfield, A table of boundary slopes of Montesinos knots, Topology 40 (2001), no. 2, 309-315. MR1808223 (2001j:57008)

[4] David Futer, Efstratia Kalfagianni, and Jessica S. Purcell, Guts of surfaces and the colored Jones polynomial, in preparation.

[5] - Symmetric links and Conway sums: volume and Jones polynomial, Math. Res. Lett. 16 (2009), no. 2, 233-253. MR2496741

[6] Stavros Garoufalidis, The Jones slopes of a knot, arXiv:0911.3627, Journal of Quantum Topology, to appear.

[7] Allen E. Hatcher, On the boundary curves of incompressible surfaces, Pacific J. Math. 99 (1982), no. 2, 373-377. MR658066 (83h:57016)

[8] Allen E. Hatcher and Ulrich Oertel, Boundary slopes for Montesinos knots, Topology 28 (1989), no. 4, 453-480. MR 1030987(91e:57016) 
[9] W. B. Raymond Lickorish, An introduction to knot theory, Graduate Texts in Mathematics, vol. 175, Springer-Verlag, New York, 1997. MR.1472978 (98f:57015)

[10] W. B. Raymond Lickorish and Morwen B. Thistlethwaite, Some links with nontrivial polynomials and their crossing-numbers, Comment. Math. Helv. 63 (1988), no. 4, 527-539. MR 966948 (90a:57010)

[11] Makoto Ozawa, Essential state surfaces for adequate knots and links, arXiv:0609166, Journal of the Australian Mathematical Society, to appear.

Department of Mathematics, Temple University, Philadelphia, Pennsylvania 19122

E-mail address: dfuter@temple.edu

Department of Mathematics, Michigan State University, East Lansing, Michigan 48824

E-mail address: kalfagia@math.msu.edu

Department of Mathematics, Brigham Young University, Provo, Utah 84602

E-mail address: jpurcell@math.byu.edu 